resulting thick wing was fully appreciated. There is an interesting discussion in the paper of an Australian project for an all-wing airliner using a 30 per cent thick wing fitted with boundary-layer suction in order to reduce the drag.

The thin swept-back wing is shown to be peculiarly susceptible to aeroelastic effects; that is, to the aerodynamic consequences of structural distortion in flight. A simple example of such an effect is the so-called aileron reversal. This is also met in unswept wings. If the extra lift produced by the downward deflexion of an aileron acts towards the rear of the wing, it produces a 'nose down' twist of the structure which will cause a net loss of lift at sufficiently high speeds. An academically interesting solution to the general problem is the 'aeroisoclinic' wing invented by G. T. R. Hill. This leads to a swept wing which is weak in torsion. Aileron reversal is to be avoided by obtaining lateral control from wing tips the incidence of which to the wind can be varied, instead of the conventional ailerons. The case for crescent wings is also made. It seems, however, that at moderate supersonic speeds there is little to beat the delta planform with its large internal volume, especially at the wing root. This allows ample space for a stiff structure, the load, the fuel, the undercarriage, and the whole variety of apparatus and equipment carried by modern aeroplanes.

It is a big problem to get highly swept wings to give adequate lift at slow speeds, and the possible solutions of the landing problem are the subject of controversy among aeronautical scientists and engineers. An American aeroplane with sweep which is variable in flight has recently been flown. There are advocates of the use of parachutes, stowed helicopter blades, etc. In fact, the shape of wings to come is as yet indeterminate because of the difficulties of flying slowly.

\section{HARNESSING KNOWLEDGE ANNUAL CONFERENCE OF ASLIB}

$T$

HE fact that Aslib could choose "The Significance of Information in Present-Day Industrial Society" as the theme of its twenty-seventh annual conference, held recently at Swanwick in Derbyshire, clearly indicates that the day is past when 'information' could be dismissed as the concern of the chemist or physicist alone. The very phrase has an Aristotelian ring that nevertheless yields nothing in emphasis to the twentieth century's pre-occupations with men as minders of machines. So, indeed, it should be, for as Sir Stephen Tallents declared in opening the conference, "ideas have to-day become not only the most potent of forces but also the most precious of cargoes". Those same ideas, communicated and applied, are the life stream of an industrialized society, which must perish unless it can produce more goods more cheaply than ever before.

In this modern industrial and scientific society, the key to future development is the speed with which new ideas can be put to work- such was the burden of the principal paper at the conference, presented by Prof. T. U. Matthew, who holds the chair of engineering production in the University of Birmingham - the only one of its kind in Great Britain. We have, he said, achieved a new type of chain-reaction in which new knowledge and ideas, travelling at a rate governed by the effectiveness of our information services and means of publication and communication, give rise to an endless succession of transmutations and further ideas in an ever-widening range of specialized fields of research and development. For the truth of this, one need not look beyond the post-war capital development programmes in coal, steel and chemical production, which have all felt the impact of modern methods of communication on a large scale.

These, it is true, are fields in which, for the most part, large industrial units are engaged. It is encouraging to know that, according to the recent survey "Research and Development in British Industry", produced by the Federation of British Industries, some $£ 30,000,000$ is now being spent on research each year compared with $£ 5,500,000$ just before the Second World War. But responsibility for bringing into the production stage the vast stores of new knowledge that become available in this way still lies generally with a comparatively small group of larger companies. Behind them stretch the thousands of firms of intermediate and smaller size, the majority of which have not, as yet, got to grips with the concept of increasing productivity, let alone the definitive shape that can be given to that concept by the utilization of published facts and figures. Add to this picture of the production side of industry the stimulus that can come from the communication of data and ideas in connexion with home and foreign markets, internal and external freight facilities, international trade and currency regulations and, not least, industrial labour relations-the vision is stirring indeed.

Here, as Prof. Matthew indicated, arise both the immediate obstacle and the long-term challenge. It is already clear, he said, that the present rate of discovery arising from fundamental research in physics, chemistry and the applied sciences, far exceeds the digestive capacity of existing development resources-hence the growing pressure for a new technological university and more technologists to bridge the gap between scientific research and industrial application. On a wider stage, the rate of exploration of the world's raw material resources has outstripped the nations' capacity to finance and organize their development. To this fact can be traced such schemes as the United Nations technical assistance projects, the 'Point 4' plan of the U.S. Government and the Colombo plan. Both nationally and internationally, these far-sighted proposals mean a mounting pressure on information services. Steps to encourage the interchange of knowledge and to speed up the processes of accumulating, abstracting, classifying and disseminating information on research and technical developments, production processes, raw material resources and market opportunities have already been taken, nationally, by Aslib, the Department of Scientific and Industrial Research and the research associations and, internationally, by the Office for European Economic Co-operation and the British Commonwealth Scientific Offices. The danger is that, in the smaller firms which have not yet established information departments of their own, these existing facilities may pass unnoticed, at least until the supply of technologists has increased. For the immediate future, he said, it is a question of assisting individual managers, who are not yet alive to the great wealth of recorded information open to them, to exploit it with only their present resources of staff. To Henri Fayol's six activities of management of fifty years ago, it is now time to add a 
seventh-the information function; and on the performance of it in the next decade or so the long-term future of British industry may well depend.

If, indeed, information services are destined to play such a crucial part in the future of British industry, much of the credit for an early realization of their potential value must go to the Royal Society's Information Services Committee and to Aslib, the work of both of which since 1948 was reviewed in papers presented by Dr. D. J. Urquhart and Mr. Leslie Wilson. The story of their achievement in barely four years is, perhaps, one of the most creditable on the less sensational side of British recovery since the War. Yet there can be no room for complacency while it can be said, as Dr. Urquhart did, that "there do not appear to be any major faults in the system [that is, of information services] as significant as the fact that so many who might use the system more, hardly use it at all". A recent survey indicated that 75 per cent of all industrial organizations have less than five periodicals of any sort and less than fifty books. At the same time, it has never been clearly demonstrated that the kind and size of firms that one might reasonably expect to find among the 75 per cent could and should be able to make use of published information on a scale likely to affect turnover materially. The firms themselves are mostly inarticulate or unaware of their need of information, technical or otherwise. The next step is surely to reduce this diffuse problem to measurable proportions, to ascertain at what level information services should operate in order to make the greatest contribution to the numerous and varied groups of potential information users. 'This is research of a kind in which, it is to be hoped, Aslib may be enabled to give an early lead.

\section{MINERAL DRESSING}

\section{SYMPOSIUM IN LONDON}

$\mathrm{A}^{\mathrm{T}}$

T the beginning of this century the extraction of valuable minerals from their host rocks and ore complexes was a craft rather than a science. With the mounting demand, first for more and more engineering metals, and later for alkali earths and the oxidized earths of the rarer metals, it became necessary to exploit low-grade ore-bodies hitherto considered uneconomical. Two World Wars accelerated industrial demand, and at the same time forced the crude processes of those days on to a more scientific basis. It is a far cry from the 'préparation mécanique' and the 'ore dressing' of the past generation to the precise process engineering of the modern mineraldressing plant. A satisfactory title for the wide range of activities embraced by this phrase 'mineral dressing' remains to be found. Treatment starts with the reduction of large pieces of rock to an average particle size of anywhere down to a few microns, and then uses suci, forces as gravity (simple or reinforced by centrifugal acceleration), magnetism and electrostatic deflexion, and, above all, the differences between the surface tension effects on minute particles in order to render them amenable to the froth flotation process and allied techniques. The field of application in industry is constantly being enlarged by the fundamental technological research work now actively in process in a number of laboratories. This again is reflected in the increasing exploitation of ore deposits hitherto considered too low in grade to be worth treating. Industrial specialization has moved mineral dressing into the position where it takes over practically everything produced underground or in open-cast work and rejects suitable quantities of valueless material, while bringing up the balance to a closely controlled specification which aims at optimum technical preparation for subsequent processes such as smelting or leaching, the production of a variety of finished substances (for example, asbestos) or the purification of such earths as phosphates to the point where wet chemical processes can best be applied to the upgraded concentrates.

Evidence of the wide scope of these technical developments was supplied by the first symposium on mineral dressing ever to take place in Great Britain, which was held during September 23-25 under the auspices of the Institution of Mining and Metallurgy at the Imperial College of Science and Technology, London, an inaugural lecture (the Sir Julius Wernher Memorial Lecture) being delivered at the Royal Institution the previous afternoon by Prof. A. M. Gaudin (see Nature, October 18, p. 656). Thirtynine papers were presented, covering the international field of research and application. Size, surface area, shape and lattice structure were brought together in a series of papers which dealt with discontinuity lattices and their effects on minerals chemically almost identical. The mathematics of crushing and grinding are at present statistical rather than fundamental, and are showing that the hypotheses of Kick and Rittinger, which during the past century have aged unobtrusively into 'laws', are not entirely satisfactory. Papers on comminution and precise measurement of products down to the $5-\mu$ range discussed the role of stresses shown at the surface of the particle as 'crack tips', and defined and described the principles and laboratory techniques now available for the measurement of sizes and surface areas of the crushed products. Sub-sieve methods using sedimentation and elutriation in gaseous and liquid columns were compared. The practical aspects of this work in its extension to process control were covered in another group of papers. One important aspect of this branch of technical research is its use in the reduction of huge tonnages of low-grade ore to a closely controlled size-range of particles having a suitably differentiated condition at their individual surfaces as a prelude to separation by froth flotation, a process which can operate in suitable cases with high efficiency on particles as small as 3 or $4 \mu$ in diameter.

The limitation of 'heavy media separation', as the process is industrially called, has been progressively reduced until to-day suitable material down to 20 mesh (Tyler) can be treated, although such a small size is exceptional. In one of the papers describing plant operation, a daily tonnage of 12,800 was mentioned, treating diamondiferous 'blue clays' down to 10 mesh by this method. The operating characteristics of the hydro-cyclone received new elucidation in two papers. This instrument applies controlled centrifugal force to a moving stream of particles and aids them to segregate in accordance with their difference of mass. Provided there is a marked difference of density in the +100 -mesh field, simple gravitational force can be used for this work ; but as the Newtonian laws of settlement give place to Stokesian sedimentation, accelerated gravitational fields must be used if large quantities are to be handled quickly. The 